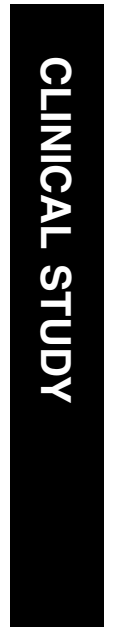

Royal Hallamshire Hospital Sheffield, UK

Correspondence:

JP Burke

Royal Hallamshire Hospital

Glossop Road

Sheffield S10 2JF, UK

Tel: + 441142712036

Fax: + 441142713682

E-mail: julie.d.taylor@

sth.nhs.uk

Received: 1 September

2001

Accepted in revised form:

19 June 2002

\section{Surgical correction of large-angle exotropia in adults}

Abstract

Aim A retrospective and longitudinal review of the outcome of strabismus surgery for adults with large- and very-large-angle manifest exodeviations, using two-, three- and four muscle horizontal recti surgery with adjustable sutures.

Methods A total of 26 consecutive adult patients undergoing surgery for socially noticeable strabismus comprising five primary, 16 consecutive, and five secondary constant exotropias with a mean near deviation of 58 prism dioptres and a mean distance deviation of $55 \Delta$ were evaluated preoperatively and at various time intervals postoperatively. Surgery involved two muscles in seven cases, three muscles in $\mathbf{1 3}$ cases, and four muscles in six cases; and 25 of 26 had adjustable sutures. There was a horizontal preoperative ocular movement deficit in $\mathbf{1 7}$ that was asymmetrical in four cases.

Results Binocularity was restored in eight patients (31\%), $20(77 \%)$ were within $10 \Delta$ of orthotropia, and $24(92 \%)$ were happy with their cosmesis. Two had symptomatic asymmetrical ocular motility deficits postoperatively following a two-muscle procedure and one required reoperation. A total of 19 patients undergoing three- or four-muscle surgery were asymptomatic postoperatively. A total of 22 patients had follow-up of 8 months or more.

Conclusion In adults with large-angle manifest exodeviations, adjustable suture surgery involving three or more horizontal recti successfully restores primary position alignment, a high degree of patient satisfaction, and can be expected to be associated with a low incidence of symptomatic postoperative asymmetrical ocular movement deficits.

Eye (2003) 17, 334-339. doi:10.1038/ sj.eye. 6700347

Keywords: exodeviation; adjustable suture; lateral incomitance; adult strabismus
ZI Currie, T Shipman and JP Burke

Introduction

Large-angle constant exotropias negatively impact on the way patients see themselves and are perceived by others. ${ }^{1}$ The benefits of surgical treatment of exodeviations in adults are well proven, not only improving a patient's psychosocial functioning, ${ }^{2,3}$ but also, in some cases, restoring binocular vision. ${ }^{4-6}$ The management of large-angle exodeviations in more recent studies falls broadly into two surgical approaches. Large bilateral lateral rectus recessions have been selected when the acuity was good in each eye, ${ }^{7-9}$ whereas a maximal or supramaximal unilateral recess-resect procedure was performed if one eye was amblyopic ${ }^{9,10}$ (sometimes also including surgery to weaken the obliques).

The disadvantage of large unilateral two-muscle procedures is the risk of inducing iatrogenic, symptomatic asymmetrical lateral incomitance.

In earlier publications, ${ }^{11,12}$ many ophthalmologists chose to operate on two horizontal muscles and correct residual deviations with second or third procedures with variable results. Others, who were in the minority, considered simultaneous four horizontal muscle surgery (without utilising adjustable sutures) to be a superior approach for these deviations. ${ }^{13,14}$

We chose to individualise our surgery to optimise primary position alignment and to minimise the production of symptomatic iatrogenic postoperative horizontally incomitant deviations. Surgical results of large unilateral or bilateral two-muscle procedures have been published previously, ${ }^{7-10}$ but data on the early and longer term postoperative outcome following surgery on three or four horizontal recti for large-angle exotropias in adults utilising adjustable sutures are scanty and imprecisely documented. This prompted us to carry out a retrospective and longitudinal study to evaluate this surgical approach. 


\section{Patients and methods}

The notes of all patients, who had strabismus surgery between July 1993 and May 1998 for exotropias measuring $45 \Delta$ or more, were examined retrospectively. Information was gathered about patient details, acuity with refractive error corrected, the preoperative and 2-week postoperative strabismus measurements and measurements at subsequent postoperative follow-up visits at 3-4, 8-12, 12-24, and 24-36 months and details of the type of surgery performed. Binocular functions were investigated and the results recorded. Measurement of the maximum angle of deviation was performed by the alternate cover and prism test, using loose prisms. The risk of symptomatic postoperative diplopia was assessed preoperatively in the majority using loose prisms in primary position or Fresnel prisms, and in a minority Botulinum toxin was also utilised. Deficits of ocular movement were measured on a five-unit scale 0 to -4 where 0 represents full or $100 \%$ abduction or adduction and -4 represents an inability to abduct or adduct beyond the midline. ${ }^{15}$ There were 26 patients: 14 males and 12 females. The mean age was 38 years (range 14-68). From the history, five patients had primary exotropias, 16 had consecutive exotropias, and five were secondary to unilateral traumatic loss of vision. The mean deviation for near was $58 \Delta$ (range 40-85) and for distance $55 \Delta$ (range 40-80). There was no evidence of binocular function demonstrated in any of the patients preoperatively.

Of the patients, 17 had a preoperative deficit of horizontal ocular movement in each eye measuring -1 or greater. This was symmetrical in 13 and asymmetrical in four (a difference in the deficit in each eye of greater than $25 \%$ or greater than 1 unit).

For patients with near-distance disparity, the target angle for surgery was generally based around the smaller angle. The number of muscles operated on was dependent on a number of factors, in particular the degree and density of amblyopia, the size of the deviation and the predicted functional significance of inducing significant lateral incomitance, previous surgery, and whether there was any abnormality of ocular movements. Peroperative forced duction testing was carried out on all patients.

The surgery involved a two-muscle procedure in seven patients, a three-muscle procedure in 13 patients, and a four-muscle procedure in six patients. Adjustable sutures were used in 25 of the 26 cases.

The goals of surgery bearing in mind known postoperative exo-drift patterns were to achieve the optimum eye alignment with one operation, to improve psychosocial functioning, and to avoid troublesome postoperative diplopia or symptomatic side-gaze incomitant deviations. For cases with significant amblyopia, we performed much or all the surgery on the amblyopic eye, provided the preoperative sensory findings indicated a low risk of symptomatic iatrogenic side-gaze postoperative diplopia.

Patient details are recorded in Table 1.

\section{Results}

Binocularity was restored in eight patients (32\%), orthophoria or a deviation of $10 \Delta$ or less for distance in $20(77 \%)$, a cosmetic result acceptable to the patient in 24 (92\%), and a reoperation rate of $4 \%$ (one patient).

Of the eight patients with binocular functions demonstrable postoperatively, three were primary exodeviations with good visual acuity who were heterophoric (Cases 1-3). Three patients with consecutive exotropias regained some binocularity (Cases 10, 17, 21) but of poor quality in two. The remaining two patients with binocularity had previous penetrating intraocular trauma. In one, vision was corrected with a contact lens (Case 26) and, in the other, with a secondary intraocular lens (Case 22).

A total of 24 patients (92\%) were happy with the cosmetic result, which was maintained at 12 months or beyond in all cases where follow-up was complete (20 of 24).

Surgical success was defined as those patients who measured $10 \Delta$ or less of heterophoria or heterotropia for distance. This was achieved in 20 cases (77\%). Of these, seven were heterophoric; the remainder had small-angle manifest eso- or exodeviations. One patient, although heterophoric for distance, had a residual $12 \Delta$ exotropia for near (Case 15).

Of the patients, 22 were followed up for at least 8-12 months postoperatively, with six having follow-up measurements 24-36 months postoperatively. Between 2 weeks and 8-12 months postoperatively, there was an average $5.5 \Delta$ exo-drift for each case. Most

(16 cases) of them demonstrated an exo-drift, while four showed an eso-shift (median 4.5 prism dioptres, range 1-8 prism dioptres) for distance viewing and two were unchanged. Of the patients with shorter follow-up, two were only seen once at a 2 -week postoperative visit. In one case (Case 12), the patient returned to his country of origin immediately after this, and in the second case (Case 25), the patient failed to attend any further followup visits (verbal communication with this patient confirms that his eyes remain well aligned 3 years after the original surgery). A third patient (Case 1) also returned to his country of origin after a 3-month followup period, and the fourth (Case 17) was happy with the surgical result at 3 months but declined further long-term follow-up. 
Table 1 Patient details (near measurements recorded above distance measurements)

\begin{tabular}{|c|c|c|c|c|c|c|c|c|}
\hline Aetiology & Age & Acuity & Preop & Surgery & Postop & 8-12 months & 18-36 months & Happy \\
\hline \multirow[t]{4}{*}{ 1. Primary XT } & 30 & $\mathrm{R} 6 / 9 \mathrm{~L} 6 / 5$ & $75 X \mathrm{~T}$ & RLRc $7 \mathrm{~mm}$ adj & $2 \mathrm{E}$ & & & Yes \\
\hline & & & & RMRs 7 mm & $5 \mathrm{RH}$ & & & \\
\hline & & & $75 X \mathrm{~T}$ & LLRc $7 \mathrm{~mm}$ adj & $9 \mathrm{E}(\mathrm{T})$ & & & \\
\hline & & & $4 \mathrm{RHT}$ & LMRs $7 \mathrm{~mm}$ & $3 R H(T)$ & & & \\
\hline \multirow[t]{3}{*}{ 2. Primary $\mathrm{XT}$} & 33 & R6/12 L6/9 & $85 X \mathrm{~T}$ & RLRc $7 \mathrm{~mm}$ adj & $6 X$ & $12 X$ & & Yes \\
\hline & & & 6RHT & RMRs $6 \mathrm{~mm}$ & $4 \mathrm{RH}$ & & & \\
\hline & & & $75 X \mathrm{~T}$ & $\begin{array}{l}\text { LLRc } 6 \mathrm{~mm} \text { adj } \\
\text { LMRs } 6 \mathrm{~mm}\end{array}$ & $2 X$ & $4 X$ & & \\
\hline \multirow[t]{4}{*}{ 3. Primary $\mathrm{XT}$} & 68 & R6/6 L6/6 & $50 X \mathrm{~T}$ & RLRc $6 \mathrm{~mm}$ adj & $2 X$ & $2 X$ & & Yes \\
\hline & & & $6 \mathrm{RHT}$ & RMRs $6 \mathrm{~mm}$ & & & & \\
\hline & & & $40 X \mathrm{~T}$ & & $2 X$ & $2 X$ & & \\
\hline & & & $2 \mathrm{RHT}$ & & & & & \\
\hline \multirow[t]{4}{*}{ 4. Primary $\mathrm{XT}$} & 48 & R6/6 L6/12 & $45 X \mathrm{~T}$ & RLRc $7 \mathrm{~mm}$ adj & $2 \mathrm{ET}$ & $4 \mathrm{XT}$ & $5 \times \mathrm{XT}$ & Yes \\
\hline & & & 8LHT & & & & & \\
\hline & & & $45 \mathrm{XT}$ & LLRc $6 \mathrm{~mm}$ & $14 \mathrm{ET}$ & $4 \mathrm{XT}$ & $5 \mathrm{XT}$ & \\
\hline & & & $6 \mathrm{LHT}$ & LMRs $5 \mathrm{~mm}$ & $4 \mathrm{LHoT}$ & & & \\
\hline \multirow[t]{4}{*}{ 5. Primary XT } & 56 & R6/5 L6/5 & 45XT & RMRc $5 \mathrm{~mm}$ & $6 \mathrm{RXT}$ & 10RXT & & Yes \\
\hline & & & 10RHoT & & 7RHoT & 8RHoT & & \\
\hline & & & 40XT & LLRc $2 \mathrm{~mm}$ adj & $8 \mathrm{RX}(\mathrm{T})$ & $12 \mathrm{RXT}$ & & \\
\hline & & & 7RHoT & LMRs $5 \mathrm{~mm}$ & 9RHoT & 8RHoT & & \\
\hline \multirow[t]{4}{*}{ 6. Consecutive } & 61 & R6/5 L6/60 & $50 X \mathrm{~T}$ & & $10-5 \mathrm{ET}$ & 3ET & $3 \mathrm{ET}$ & No \\
\hline & & & & & 3RHT & 2LHoT & 2LHoT & \\
\hline & & & $40 X \mathrm{~T}$ & LLRc $6 \mathrm{~mm}$ adj & $15 \mathrm{ET}$ & 14LET & 14LET & \\
\hline & & & 3RHT & LMRs/adv $7 \mathrm{~mm}$ & $4 \mathrm{RHT}$ & $2 \mathrm{LHoT}$ & $4 \mathrm{LHoT}$ & \\
\hline \multirow[t]{4}{*}{ 7. Consecutive } & 37 & R6/4 L6/24 & $65 X \mathrm{~T}$ & RLRc 7 mm adj & $6 \mathrm{ET}$ & 4LET & & Yes \\
\hline & & & $8 \mathrm{LHT}$ & RMRs $5 \mathrm{~mm}$ & & $4 \mathrm{LHT}$ & & \\
\hline & & & $65 X \mathrm{~T}$ & LLRc $7 \mathrm{~mm}$ adj & 2ET & $4 \mathrm{LHT}$ & & \\
\hline & & & 8RHoT & LMRs $5 \mathrm{~mm}$ & 2LHT & & & \\
\hline \multirow[t]{3}{*}{ 8. Consecutive } & 34 & R CF L6/5 & $60 X \mathrm{~T}$ & RLRc $11 \mathrm{~mm}$ & $25 \mathrm{ET}$ & $25 \mathrm{ET}$ & & No \\
\hline & & & & RMRs/adv $9 \mathrm{~mm}$ & & & & \\
\hline & & & $65 \times \mathrm{T}$ & & $30 \mathrm{ET}$ & $25 \mathrm{ET}$ & & \\
\hline \multirow[t]{4}{*}{ 9. Consecutive } & 50 & R6/6 L6/24 & $85 X \mathrm{~T}$ & RLRc $7 \mathrm{~mm}$ adj & $13 \mathrm{ET}$ & $16 \mathrm{ET}$ & 20LET & Yes \\
\hline & & & 15RHT & RMRs $7 \mathrm{~mm}$ adj & 2RHT & 10RHT & 8LHoT & \\
\hline & & & $75 X \mathrm{~T}$ & LLRc $7.5 \mathrm{~mm}$ adj & $16 \mathrm{ET}$ & 10LET & 30LET & \\
\hline & & & 15RHT & LMRs/adv $7.5 \mathrm{~mm}$ & $6 \mathrm{RHT}$ & $5 \mathrm{RH}$ & $10 \mathrm{LHoT}$ & \\
\hline \multirow[t]{2}{*}{ 10. Consecutive } & 24 & $\mathrm{R} 6 / 5 \mathrm{~L} 6 / 6$ & $65 X \mathrm{~T}$ & RLRc $6 \mathrm{~mm}$ adj & $6 \mathrm{X}$ & $3 \mathrm{E}(\mathrm{T})$ & & Yes \\
\hline & & & $55 \times \mathrm{T}$ & $\begin{array}{l}\text { LMRc } 6 \mathrm{~mm} \text { adj } \\
\text { LMRs } 6 \mathrm{~mm}\end{array}$ & $10 \mathrm{ET}$ & $16 \mathrm{ET}$ & & \\
\hline \multirow[t]{3}{*}{ 11. Consecutive } & 32 & $\mathrm{R} 6 / 4 \mathrm{~L} 2 / 60$ & $65 X \mathrm{~T}$ & LLRc $9 \mathrm{~mm}$ adj & $6 \mathrm{ET}$ & Straight & $4 \mathrm{XT}$ & Yes \\
\hline & & & & LMRs/adv $8.5 \mathrm{~mm}$ & & & & \\
\hline & & & $65 X \mathrm{~T}$ & & $5 \mathrm{ET}$ & $10 X T$ & $16 \mathrm{XT}$ & \\
\hline \multirow[t]{4}{*}{ 12. Consecutive } & 44 & R6/5 L6/60 & $55 \mathrm{XT}$ & LLRc $7.5 \mathrm{~mm}$ adj & $10 X \mathrm{~T}$ & & & Yes \\
\hline & & & 7LHoT & LMRs/adv $9 \mathrm{~mm}$ & & & & \\
\hline & & & $65 X \mathrm{~T}$ & & $5 \times \mathrm{XT}$ & & & \\
\hline & & & 7RHT & & & & & \\
\hline \multirow[t]{4}{*}{ 13. Consecutive } & 32 & $\mathrm{R} 6 / 4 \mathrm{~L} 6 / 4$ & $55 \times \mathrm{T}$ & RLRc $8 \mathrm{~mm}$ adj & $4 \mathrm{LET}$ & 6LET & & Yes \\
\hline & & & & & & 3LHoT & & \\
\hline & & & $50 X \mathrm{~T}$ & LLRc $8 \mathrm{~mm}$ & $2 \mathrm{ET}$ & 5LET & & \\
\hline & & & & LMRs/adv $5 \mathrm{~mm}$ & & $2 \mathrm{LHoT}$ & & \\
\hline \multirow[t]{4}{*}{ 14. Consecutive } & 54 & $\mathrm{R} 6 / 36 \mathrm{~L} 6 / 5$ & $60 X T$ & RLRc $8 \mathrm{~mm}$ adj & $3 \mathrm{ET}$ & $6 \mathrm{ET}$ & & Yes \\
\hline & & & & RMRs 6 mm & & & & \\
\hline & & & $60 X \mathrm{~T}$ & LLRc 8 mm & $10 \mathrm{ET}$ & $4 \mathrm{ET}$ & & \\
\hline & & & & & 2RHT & & & \\
\hline \multirow[t]{4}{*}{ 15. Consecutive } & 38 & $\mathrm{R} 6 / 5 \mathrm{~L} 6 / 5$ & $55 \times \mathrm{T}$ & RLRc $8 \mathrm{~mm}$ adj & $12 \mathrm{XT}$ & $12 \mathrm{LXT}$ & & Yes \\
\hline & & & & RMRs $6 \mathrm{~mm}$ & $8 \mathrm{LHT}$ & $6 \mathrm{LH}$ & & \\
\hline & & & $45 X \mathrm{~T}$ & LMRs/adv $10 \mathrm{~mm}$ & $4 \mathrm{ET}$ & $2 X$ & & \\
\hline & & & $4 \mathrm{RHT}$ & & 2LHT & $5 \mathrm{LH}$ & & \\
\hline 16. Consecutive & 32 & $\mathrm{R} 6 / 4 \mathrm{~L} 6 / 5$ & $55 \mathrm{XT}$ & LLRc $6.5 \mathrm{~mm}$ adj & $4 \mathrm{ET}$ & $4 \mathrm{XT}$ & & Yes \\
\hline & & & 20LHT & LMRs $5 \mathrm{~mm}$ & $35 \mathrm{LHT}$ & 25LHT & & \\
\hline & & & 55XT44 & & 2ET & $2 \mathrm{XT}$ & & \\
\hline & & & $12 \mathrm{LHT}$ & & 30LHT & 20LHT & & \\
\hline
\end{tabular}


Table 1 (continued)

\begin{tabular}{|c|c|c|c|c|c|c|c|c|}
\hline Aetiology & Age & Acuity & Preop & Surgery & Postop & 8-12 months & 18-36 months & Happy \\
\hline \multirow[t]{4}{*}{ 17. Consecutive } & 17 & R6/6 L6/6 & 40XT & RMRs/adv $4.5 \mathrm{~mm}$ & $4 \mathrm{ET}$ & & & Yes \\
\hline & & & $\begin{array}{l}4 \mathrm{RHT} \\
45 \mathrm{TT}\end{array}$ & & & & & \\
\hline & & & 45XT & LLKc $5 \mathrm{~mm}$ ad] & $6 \mathrm{E} 1$ & & & \\
\hline & & & 3RHT & LMRs/adv $6 \mathrm{~mm}$ & & & & \\
\hline \multirow[t]{4}{*}{ 18. Consecutive } & 56 & R6/4 L6/18 & $55 \mathrm{XT}$ & LLRC $5.5 \mathrm{~mm}$ adj & $2 \mathrm{XT}$ & $4 \mathrm{ET}$ & & Yes \\
\hline & & & 6LHT & LMRs 6 mm & & & & \\
\hline & & & $45 X \mathrm{~T}$ & & 3ET & $2 \mathrm{XT}$ & & \\
\hline & & & 2LHT & & & & & \\
\hline \multirow[t]{4}{*}{ 19. Consecutive } & 28 & $\mathrm{R} 6 / 4 \mathrm{~L} 6 / 4$ & $65 X \mathrm{XT}$ & RLRc $6 \mathrm{~mm}$ adj & 2ET & $2 \mathrm{XT}$ & & Yes \\
\hline & & & 2RHT & RMRs/adv $5 \mathrm{~mm}$ & 2LHoT & 7LhoT & & \\
\hline & & & $60 X \mathrm{~T}$ & LLRc 6 mm adj & 3ET & $5 \mathrm{XT}$ & & \\
\hline & & & 2RHT & LMRs/adv $4 \mathrm{~mm}$ & 8LHoT & 7LHoT & & \\
\hline \multirow[t]{4}{*}{ 20. Consecutive } & 29 & R6/5 L6/5 & 45XT & RLRc $7 \mathrm{~mm}$ adj & 3ET & & $2 \mathrm{ET}$ & Yes \\
\hline & & & 12 RHoT & & RHoT & & RHoT & \\
\hline & & & 45XT & LLRc $6 \mathrm{~mm}$ & $8 \mathrm{ET}$ & & $4 \mathrm{XT}$ & \\
\hline & & & 15 RHoT & LMRs $5 \mathrm{~mm}$ & RHoT & & RHoT & \\
\hline \multirow[t]{4}{*}{ 21. Consecutive } & 14 & R6/6 L6/4 & 40XT 5 & RLRc 4 mm adj & $14 \mathrm{X}$ & $4 \mathrm{E}(\mathrm{T})$ & & Yes \\
\hline & & & RHoT & RMRs/adv $3.5 \mathrm{~mm}$ & & $4 \mathrm{RH}$ & & \\
\hline & & & $50 X \mathrm{~T}$ & LMRs/adv 6 mm & $7 X$ & $1 \mathrm{E}$ & & \\
\hline & & & $4 \mathrm{RHT}$ & & $3 \mathrm{RH}$ & $3 \mathrm{RH}$ & & \\
\hline \multirow[t]{2}{*}{ 22. Trauma } & 41 & R6/5 L6/12 & $80 \mathrm{XT}$ & $\begin{array}{l}\text { RLRc } 7 \mathrm{~mm} \text { adj } \\
\text { RMRs } 5 \mathrm{~mm}\end{array}$ & 14LET & $7 X(\mathrm{~T})$ & $1 \mathrm{ET}$ & Yes \\
\hline & & & $70 X \mathrm{~T}$ & $\begin{array}{l}\text { LLRc } 7 \mathrm{~mm} \\
\text { LMRs } 5.5 \mathrm{~mm}\end{array}$ & 18LET & $8 X(\mathrm{~T})$ & $1 \mathrm{ET}$ & \\
\hline \multirow[t]{2}{*}{ 23. Trauma } & 26 & R6/6 L6/9 & $55 \times \mathrm{T}$ & $\begin{array}{l}\text { RLRc } 5.5 \mathrm{~mm} \text { adj } \\
\text { RMRs } 5 \mathrm{~mm}\end{array}$ & $8 \mathrm{XT}$ & $6 \mathrm{XT}$ & & Yes \\
\hline & & & $45 X \mathrm{~T}$ & LLRc 8 mm & $2 \mathrm{ET}$ & $6 \mathrm{XT}$ & & \\
\hline \multirow[t]{4}{*}{ 24. Trauma } & 33 & $\mathrm{R} 6 / 24 \mathrm{~L} 6 / 4$ & $60 X \mathrm{~T}$ & RLRc $8 \mathrm{~mm}$ & 6RHo & & $4 \mathrm{XT}$ & Yes \\
\hline & & & 10RHoT & & & & & \\
\hline & & & $50 X \mathrm{~T}$ & LLRc $6.5 \mathrm{~mm}$ adj & $4 \mathrm{E}$ & & $2 \mathrm{ET}$ & \\
\hline & & & 10RHoT & LMRs $5 \mathrm{~mm}$ & 6RHoT & & & \\
\hline \multirow[t]{4}{*}{ 25. Trauma } & 34 & R6/4 L6/18 & $52 X \mathrm{~T}$ & RLRc $7 \mathrm{~mm}$ adj & $3 \mathrm{E}(\mathrm{T})$ & & & Yes \\
\hline & & & & & $\mathrm{RH}(\mathrm{T})$ & & & \\
\hline & & & $50 X \mathrm{~T}$ & LLRc 7 mm & $3 X$ & & & \\
\hline & & & 4RHT & LMRs 6 mm & $5 \mathrm{RH}(\mathrm{T})$ & & & \\
\hline \multirow[t]{4}{*}{ 26. Trauma } & 42 & R6/4 L6/6 & $40 X \mathrm{~T}$ & RLRc $7 \mathrm{~mm}$ adj & 10ET & $2 X$ & & Yes \\
\hline & & & 18LHT & RMRs 5 mm & 20LHT & $2 \mathrm{LH}$ & & \\
\hline & & & $45 \mathrm{XT}$ & LLRc 7 mm & 10ET & $2 \mathrm{E}$ & & \\
\hline & & & 2LHoT & & 12LHoT & $2 \mathrm{LH}$ & & \\
\hline
\end{tabular}

Note: X, exophoria; XT, exotropia; X(T), exophoria sometimes manifest; E, esophoria; ET, esotropia; E(T), esophoria sometimes manifest; H, hyperphoria; HT, hypertropia; H(T), hyperphoria sometimes manifest; RLRc, right lateral rectus recession; LLRc, left lateral rectus recession; RMRs, right medial rectus resection; LMRs, left medial rectus resection; Min, minimum; adv, muscle advanced; adj, adjustable suture; HoT, hypotropia. B.S.V Stereoacuity: Cases 1, $200^{\prime \prime} ; 2,240^{\prime \prime} ; 3,50^{\prime \prime} ; 10$ and 23 Baggolini; $17,3000^{\prime \prime} ; 21,600^{\prime \prime} ; 22,400^{\prime \prime} ; 26,200^{\prime \prime}$.

Six cases fell outside our objective criteria for surgical success. Four had esotropias and two residual exotropias. Of the exotropias, one measured $12 \Delta$ exotropia for distance at 1 year (Case 5), and the other measured $4 \Delta$ exotropia at near and $16 \Delta$ exotropia at distance at 24 months (Case 11). Of those with esotropia postoperatively, one measured $3 \Delta$ intermittent esotropia at near with sensory fusion and $16 \Delta$ esotropia at distance. One patient with a preoperative angle of $85 \Delta$ measured $16 \Delta$ esotropia immediately postoperatively, but increased to $30 \Delta$ esotropia 2 years later. She was still happy with the cosmetic appearance and discharged.

Overall, 17 patients had an initial postoperative esotropia of whom there was some exo-drift in eight with time. Of the 17 patients, 13 were within $10 \Delta$ of orthotropia long term and four were outside this criterion, but this was due to exo-drift in only one case.

Two patients were unhappy with the initial cosmetic outcome. Both patients had consecutive esotropias. One had a postoperative esotropia which remained static, measuring $25 \Delta$ for distance and near at 12 months (Case 8 ). This was the only patient who refused surgery utilising adjustable sutures and requested surgery on the densely amblyopic eye. She had a preoperative adduction deficit of -2 in the amblyopic eye that became a -3 abduction deficit following a large two-muscle procedure on this eye and required reoperation. 
The second had a $3 \Delta$ esotropia for near and $14 \Delta$ esotropia for distance that remained static at 3 years postoperatively (Case 6). The patient had a preoperative adduction deficit of -3 in the amblyopic eye, which became a -2 abduction deficit following two-muscle surgery on this eye. Although her primary position angle remained small and her cosmesis in primary good, the asymmetric incomitant deviation appears to have contributed to the patient's dissatisfaction. Further surgery was not undertaken.

Some postoperative abduction deficit was noted in 13 patients (50\%), of whom symmetrical small deficits of -1 or less were seen in five, and small asymmetrical deficits of -1 or less in four. None of these nine patients had any symptoms attributable to their abduction deficits. Four cases had larger, asymmetrical abduction deficits. These included the two patients dissatisfied with their cosmetic appearance. In the other two cases, measurements were taken at their only follow-up visits 2 weeks postoperatively. It is likely that swelling and discomfort contributed to the movement deficits, as a similar picture was seen in many of the other cases, but these deficits resolved or reduced with time.

Five out of the seven patients with two-muscle procedures had asymmetrical abduction deficits although only symptomatic in the two cases mentioned above. None of the patients with three or more muscle procedures had any asymmetry of horizontal extraocular movement postoperatively.

\section{Discussion}

The definition of a successful outcome depends entirely on the criteria used for such success. Many define satisfactory alignment as within 10 prism dioptres of orthotropia, ${ }^{7,8,14}$ while others extend the criteria to within 15. ${ }^{9,10}$

Velez ${ }^{10}$ reported a $40 \%$ success rate (within $15 \Delta$ ) for correction of large-angle ( $50 \Delta$ and greater) exodeviations in amblyopic eyes using a unilateral recess/resect procedure. This improved to $80 \%$ when combined with weakening of the inferior and superior obliques, but his paper makes no mention of postoperative ocular movement deficits. Schwartz and Calhoun ${ }^{9}$ described 22 patients without preoperative lateral incomitance who had two-muscle procedures (14 underwent bilateral lateral rectus recessions; eight underwent unilateral recession/resection) for exotropias ranging from $45 \Delta$ to $85 \Delta$ with recessions ranging from 8 to $14 \mathrm{~mm}$. Their overall surgical success rate to within $15 \Delta$ of orthophoria was $77 \%$. They described postoperative unilateral abduction deficits, some large and ranging between 3 and $6 \mathrm{~mm}$ of temporal scleral show on attempted abduction in patients with unilateral recess-resect procedures, which they stated were asymptomatic.

Berland $e t a l^{7}$ reported on a series of 30 patients with bilateral large lateral rectus recessions of $8-9 \mathrm{~mm}$ for deviations measuring from $35 \Delta$ to $65 \Delta$. They noted a success rate of $43 \%$ within $10 \Delta$ of orthophoria and a reoperation rate of $20 \%$. They detected a small abduction deficit in $30 \%$ of patients.

In our series, $77 \%$ of cases (20 patients) measured within $10 \Delta$ of orthotropia for distance. If success was defined as within $15 \Delta$ of orthotropia, then the success rate increased to $85 \%$ (21 patients). Four cases (15\%) measured more than $15 \Delta$ of eso- or exotropia and could be considered failures although one case demonstrated sensory fusion and only one of these patients was unhappy with their primary position postoperative alignment. The ultimate determinant of success is subjective patient satisfaction ( $92 \%$ in this series with one operation; 24 patients). This series also shows that patients can be very satisfied with residual deviations greater than $10 \Delta$ on either side of orthotropia and that residual deviations can appear considerably less and look better cosmetically than would be predicted from the objective deviation measured by the alternate cover and prism test.

An abduction deficit postoperatively is frequently desirable and generally asymptomatic, particularly when symmetrical. Asymmetrical deficits are more likely to be symptomatic owing to asymmetric side-gaze diplopia or to poor side-gaze cosmesis. Our series confirms the findings of Schwartz and Calhoun ${ }^{9}$ that two-muscle surgery for large-angle exodeviations on the amblyopic eye produces asymmetrical abduction deficits with the potential for these to be symptomatic. In contrast, our data demonstrated that spreading the surgery over three or four of the horizontal recti produces a high degree of side-gaze ocular movement symmetry even where there is significant preoperative asymmetry. In patients with constant large-angle exodeviations where the psychosocial consequences of the exotropia are the main indication for seeking surgery, the aim is to optimise eye alignment with the minimum number of surgical interventions. We would emphasise the benefits of individualised one-stage surgery, using all four horizontal recti if necessary. Our results show that as an alternative to large bilateral lateral rectus recessions where the documented reoperation rates are higher and supramaximal unilateral recess/resect surgery where asymmetric side-gaze incomitance is higher, three and four horizontal muscle surgery can produce postoperative outcomes, even in these very large-angle exodeviations comparable to those achieved for smaller angle deviations. In addition, the latter minimises the 
potential subjective complications of asymmetrical horizontal ocular movement deficits.

\section{References}

1 Olitski SE, Sudesh S, Graziano A, Hamblen J, Brooks SE, Shaha $\mathrm{SH}$. The negative psychosocial impacts of strabismus in adults. JAAPOS 1999; 3: 209-211.

2 Satterfield D, Keltner J, Morrison T. Psychological aspects of strabismus study. Arch Ophthalmol 1993; 111: 1100-1105.

3 Burke JP, Leach CM, Davis H. Psychosocial implications of strabismus surgery in adults. J Pediatr Ophthalmol Strabismus 1997; 34: 159-164.

4 Goldstein JH, Schneekloth BB. The potential for binocular vision in constant exotropia. Am Orthopt J 1993; 43: 67-70.

5 Morris RJ, Scott WE, Dickey CF. Fusion after surgical alignment of longstanding strabismus in adults. Ophthalmology 1993; 100: 135-138.

6 Ball A, Drummond GT, Pearce WG. Unexpected stereoacuity following surgical correction of long-standing horizontal strabismus. Can J Ophthalmol 1993; 5: 217-220.
7 Berland JE, Wilson ME, Saunders RA. Results of large (8-9 $\mathrm{mm}$ ) bilateral lateral rectus recessions for exotropia. Binocular Vision Strabismus Q 1998; 13: 97-104.

8 Stoller SH, Simon JW, Lininger LL. Bilateral lateral rectus recession for exotropia: a survival analysis. J Pediatr Ophthalmol Strabismus 1994; 31: 89-92.

9 Schwartz RL, Calhoun JH. J Pediatr Ophthalmol Strabismus 1980; 17: 359-363.

10 Velez G. Surgical treatment of exotropia with poor vision. Strabismus II. Proceedings of the Fourth Meeting of the International Strabismological Association, 1984, pp 263-267.

11 Burian H, Spivey B. The surgical management of exodeviations. Trans Am Ophthalmol Soc 1964; 62: 276-284.

12 Mulberger R, McDonald P. Surgical management of nonparalytic exotropia. Arch Ophthalmol 1954; 52: 664.

13 Azar RF. Surgical management of exotropia exceeding 70 prism diopters. Ann Ophthalmol 1971; 3: 159-166.

14 Urist MJ. Right-angle exotropia. Am J Ophthalmol 1964; 58: 987-1004.

15 Doughty DD, Lennarson LW, Scott EW. Perspect Ophthalmol 1978; 2: 55-59. 